\title{
miR-383 inhibits ovarian cancer cell proliferation, invasion and aerobic glycolysis by targeting LDHA
}

\author{
R. L. HAN ${ }^{1}$, F. P. WANG ${ }^{1}$, P. A. ZHANG ${ }^{1}$, X. Y. ZHOU², Y. LI',* \\ ${ }^{1}$ Department of Clinical Laboratory, Renmin Hospital of Wuhan University, Wuhan, People's Republic of China; ${ }^{2}$ Department of Cardiology, \\ Renmin Hospital of Wuhan University, Wuhan, People's Republic of China.
}

*Correspondence: yanlitf1120@163.com, zhouchuanyiyy@163.com

Received September 11, 2016 / Accepted October 22, 2016

\begin{abstract}
MicroRNAs (miRNAs) are differentially expressed in various cancers and act as oncogenes or tumor suppressors. MiR383 has been characterized as a cancer suppressor in several cancers. However, the exact expression patterns of miR-383 and the precise molecular mechanisms underlying its role in ovarian cancer have not been investigated thoroughly. In this study, we found that the expression of miR-383 was significantly downregulated in ovarian cancer tissues and ovarian cancer cell lines. Ectopic expression of miR-383 remarkably suppressed the ovarian cancer cell proliferation by enhancing cell apoptosis and significantly inhibited the invasion of ovarian cancer cells, while low expression of miR-383 exhibited the opposite effect. Bioinformatics analysis suggested LDHA as a novel target of miR-383, and miR-383 suppressed the expression level of LDHA mRNA by direct binding to its 3'-untranslated region (3'UTR). Expression of miR-383 was negatively correlated with LDHA in ovarian cancer tissues. In addition, modulation of miR-383 expression could affect the aerobic glycolysis in the ovarian cancer cells. Furthermore, Silencing of LDHA counteracted the effects of miR-383 suppression, while its overexpression reversed tumor inhibitory effects of miR-383. In conclusion, our study demonstrated that miR-383 regulated LDHA expression in ovarian cancer cells, thereby stunting glycolysis, cell proliferation and invasion.
\end{abstract}

Key words: ovarian cancer, LDHA, miR-383, aerobic glycolysis

Ovarian cancer is the most lethal gynecological malignancy and ranks the fifth in the cause of cancer-related death among women world-wide [1]. Despite improvements in the diagnosis and treatment of this cancer, the mortality rate of ovarian cancer has been only modestly changed over the last decade. For a lack of specific early symptoms, it is often diagnosed at an advanced end-stage and often metastasizes to distant organs, leading to a high mortality and low 5-year survival rate [2]. Therefore, it is important to elucidate the molecular mechanisms underlying the development and progression of ovarian cancer, which will provide crucial insights to discover novel diagnostic biomarkers and therapeutic approaches for ovarian cancer patients.

MicroRNAs are a class of small, non-coding RNAs of 20-24 nucleotides in length that negatively regulate gene expression at the post-transcriptional level through base pairing with the 3'UTR of the target mRNA, leading to target mRNA degradation and/or translational repression [3]. Accumulating evidence has confirmed that miRNAs are frequently dysregu- lated in various human cancers and act either as oncogenes or tumor suppressors owing to their critical roles in diverse biological functions, such as cell proliferation, apoptosis as well as differentiation [4]. To date, large number of miRNAs has been implicated in the development and progression of ovarian cancer [5-7]. MiR-383 has been reported to serve as a tumor suppressor and is downregulated in several cancer types, such as esophageal squamous carcinoma, hepatocellular carcinoma, pancreatic carcinoma and glioma [8-11]. Numerous studies have demonstrated that miR-383 is involved in the ovarian development and a recent microRNA microarray analysis found that miR-383 was significantly underexpressed in ovarian cancer [12-14]. Thus, it would be of interest to investigate the functional roles and the exact mechanisms of miR-383 involved in ovarian carcinogenesis.

Reprogrammed cellular metabolism is a major feature of cancer [15]. The aerobic glycolysis, known as Warburg effect, describes a common phenomenon by which cancer cells preferentially utilize glucose through glycolysis over oxida- 
tive phosphorylation regardless of their oxygen status [16]. This metabolic shift toward enhanced glycolysis is believed to provide metabolic needs for the rapid proliferating cancer cells to grow, rather than energy production. Lactate dehydrogenase A (LDHA) is a step-control enzyme which has been identified to play critical roles in regulation of the glycolysis pathway [17]. Accumulating evidence has shown that LDHA were upregulated and correlates with the clinicopathologic features and prognosis of patients in many types of human cancers [18-20]. Several recent studies have shown that LDHA is involved in the growth of ovarian cancer [21-23]. However, the mechanisms that underlie epigenetic regulation of LDHA expression in ovarian cancer remain unclear.

In the present study, we demonstrated that miR-383 is significantly downregulated in human ovarian cancer tissue and cell lines. Ectopic expression of miR-383 suppressed cell proliferation and invasion via decreasing glycolysis by directly targeting LDHA, and miR-383 expression is negatively correlated with LDHA expression in ovarian caner. These findings suggest that the miR-383/LDHA axis plays a vital role in ovarian cancer progression through acting as an important regulator of glucose metabolism.

\section{Materials and methods}

Clinical samples, cell lines and transfection. Ovarian cancer tissues and matched adjacent normal tissues were obtained from 24 patients with ovarian cancer who received surgery at the Wuhan Tumor Hospital, Wuhan, China. Samples were obtained with informed consent, and the study had been approved by local institutional review boards on human subject research and in accordance with the Declaration of Helsinki. All the histological diagnoses for ovarian cancer and normal tissues were reviewed and recognized by two pathologists independently. Sample characteristics are described in Table1. HOSEpiC (the normal ovarian surface epithelial cell line) and five human cervical cancer cell lines SKOV3, ES-2, OVCAR-3, Caov-3 and COC1 were obtained from Chinese Center for Type Culture Collection (Wuhan, China) and cultured according to the provider's instruction. Transfection was performed when cells were grown to $70 \%$ confluence, using the Lipofectamine 2000 (Invitrogen, Carlsbad, CA, USA) according to the manufacturer's instructions.

MicroRNA and plasmid construction. MiR-383 mimics/ inhibitor and corresponding controls were purchased from RiboBio (Guangzhou, China). LDHA-specific siRNA and negative control were got from Ambion (Shanghai, China). LDHA cDNA was subcloned into pcDNA3.1 to construct the LDHA expression vector. For luciferase reporter, the 3'UTR of LDHA containing the putative binding sites for miR-383 was amplified by PCR from human genomic DNA using the following forward primer: 5 '-gtcatctagaCAGACCTTGTGAAGGTGACTCTG-3' and the reverse primer: 5'-gtcatctagaCCAGCTTCCAGAGGACAAGATCTC-3', both of which contain a Xbal restriction site. The PCR product was digested with $\mathrm{XbaI}$ and subcloned into pGL3 luciferase reporter plasmid (Promega, Madison, WI, USA). Mutations in the miR-383 binding site of LDHA 3'UTR were produced by the QuikChange Site-Directed Mutagenesis Kit (Stratagene, La Jolla, CA, USA). Constructs were confirmed by sequencing.

RNA extraction and qRT-PCR. Total RNA and miRNA were extracted from tissues and cells using RNeasy Mini and miRNeasy Mini Kits (Qiagen, Valencia, CA, USA) according to the manufacturer's instructions. For investigation of miR-383 expression, stem-loop RT-PCR was performed as described [24]. Expression of U6 was used as an endogenous control. Real-time PCR was performed using FastStart Universal SYBR Green Master kit (Roche Diagnostics, Mannhelm, Germany) and analyzed with an Applied Biosystems 7900 Real-Time PCR System. Primer sequences were as follows: LDHA, 5'-TGGAGTGGAATGAATGTTGC-3' and 5 -ATAGCCCAGGATGTGTAGCC-3'; and $\beta$-actin, 5'-GATCATTGCTCCTCCTGAGC-3' and 5'-ACTCCTGCTTGCTGATCCAC-3'. Fold changes in expression were calculated. The qRT-PCR data was analysed using the method of $2^{-\Delta \Delta \mathrm{Ct}}$ relative expression quantity as previously described [25]. All the qRT-PCRs were run in triplicate.

Cell proliferation, cell cycle analysis and apoptosis assay. Transfected cells were planted into 96 -well plates $\left(2 \times 10^{3} /\right.$ well) and cultured for 0, 24, 48, 72 and $96 \mathrm{~h}$. MTT (5 mg/ $\mathrm{ml}$ ) was added to each well for $4 \mathrm{~h}$ at $37^{\circ} \mathrm{C}$. The reaction was stopped by $150 \mu \mathrm{DMSO}$, and absorbance readings at $490 \mathrm{~nm}$ were obtained in triplicate using a spectrophotometric plate reader (Thermo Scientific, Waltham, MA, USA). For the cell

Table 1. Clinicopathologic characteristics of ovarian cancer patients

\begin{tabular}{lc}
\hline Clinicopathological parameters & Cases $(\mathrm{n}=24)$ \\
\hline Age (years) & 14 \\
$\quad \leq 55$ & 10 \\
$>55$ & \\
FIGO stage & 8 \\
$\quad$ I-II & 16 \\
III-IV & \\
Tumor size $(\mathrm{cm})$ & 9 \\
$<5$ & 15 \\
$\geq 5$ & \\
Grade & 10 \\
$1 / 2$ & 14 \\
3 & \\
Histological type & 20 \\
Serous & 4 \\
Others & \\
Peritoneal metastasis & 14 \\
Yes & 10 \\
No & \\
Lymph node metastasis & 3 \\
Yes & 21 \\
No &
\end{tabular}


cycle analysis, cells were harvested by trypsinization, washed twice using cold PBS and fixed in 70\% ethanol overnight at $4{ }^{\circ} \mathrm{C}$. Then, cells were subsequently incubated with $20 \mu \mathrm{g} / \mathrm{ml}$ propidium iodide (Sigma, St. Louis, MO, USA) for $20 \mathrm{~min}$ at room temperature, and cell cycle analysis was performed with FACS flow cytometry (BD Biosciences, Franklin Lakes, NJ, USA). For the cell apoptosis analysis, cells with different treatment were seeded in six-well plates at $3 \times 10^{5}$ cells per well in the absence of FBS for $48 \mathrm{~h}$. Then, cells were harvested and labeled with FITC-conjugated Annexin V and propidium iodide (BD Pharmingen) following the manufacturer's instructions. The apoptotic cells were measured by FACS.

Transwell invasion assay. Cell invasion assay was performed with Matrigel-coated transwell cell culture chambers (Millipore, Billerica, MA, USA). Aliquots of cells $\left(1 \times 10^{5}\right)$ were placed into upper chambers coated with $150 \mathrm{mg}$ Matrigel (BD Biosciences, Bedford, MD, USA). The lower chambers were filled with DMEM containing 10\% FBS. After incubation at $37^{\circ} \mathrm{C}$ for $24 \mathrm{~h}$, cells remaining on the upper surface of the membrane were removed. Cells on the lower surface of the membrane were fixed and stained with crystal violet. The invaded cells were counted at $\times 200$ magnification from five random fields of each filter. The assays were performed in triplicate and were repeated three times.

Dual-luciferase assay. For the dual-luciferase assay, HEK293 cells in a 96-well plate were transfected with $50 \mathrm{nM} \mathrm{miR-383} \mathrm{or}$ miR-NC. The cells were then cotransfected with $0.2 \mathrm{mg} / \mathrm{ml}$ of vector with the wild-type or mutant 3'UTR of LDHA gene. After $48 \mathrm{~h}$, luciferase activity was measured with the Dual-Luciferase Reporter Assay System (Promega). Firefly luciferase activity was then normalized to the corresponding Renilla luciferase activity. Luciferase assays were performed in quadruplicate and repeated in three independent experiments.

Western blot. Proteins were extracted by RIPA lysis buffer (Beyongtime, China). Protein concentrations were quantified by the BCA protein assay kit (Beyotime, Haimen, China). Equal amounts of protein were separated by SDS-PAGE, transferred onto PVDF membranes (Bio-Rad, Hercules, CA, USA) and blocked for $0.5 \mathrm{~h}$ at room temperature. Membranes were probed with primary antibodies against LDHA and $\beta$-actin (Santa Cruz Biotechnology, Santa Cruz, CA, USA) at $4^{\circ} \mathrm{C}$ overnight followed by incubation with HRP-conjugated secondary antibodies. Blots were detected using an ECL detection system.

Analysis of lactate production, glucose uptake, ATP level and Oxygen consumption in cells. Lactate production was measured by the lactate assay kit (BioVision, Mountain View, USA) following the manufacturer's protocol. Glucose uptake was examined through 2-NBDG (Cayman Chemicals, Michigan, USA) according to manufacturer's instruction. Fluorescent intensities were calculated using Image J software. ATP levels were tested by a CellTiter-Glo ${ }^{\circ}$ Luminescent Cell Viability Assay Kit (Promega, Madison, WI, USA). Oxygen consumption (OCR), a measure of oxidative metabolism was performed by the SeaHorse XF24 Extracellular Analyzer from SeaHorse Bioscience (Billerica, MA). SeaHorse XF24 Extracellular Analyzer was run using 8 minute cyclic protocol commands (mix for 3 minutes, let stand 2 minutes, and measure for 3 minutes).

Statistical analysis. SPSS16.0 statistical software package (SPSS, Chicago, IL, USA) was used for statistical analysis. Experiments were repeated independently at least three times, and the results are expressed as mean \pm SD. The correlation between miR-383 and LDHA was analyzed using Spearman's correlation test. Statistical differences between groups were evaluated using Student's paired two-tailed t-test. $\mathrm{P}<0.05$ was considered statistically significant.

\section{Results}

MiR-383 is downregulated in ovarian cancer clinical samples and cell lines. A previous microarray-based study showed that miR-383 was likely downregulated in ovarian cancer. To further confirm the aberrant expression of miR-383
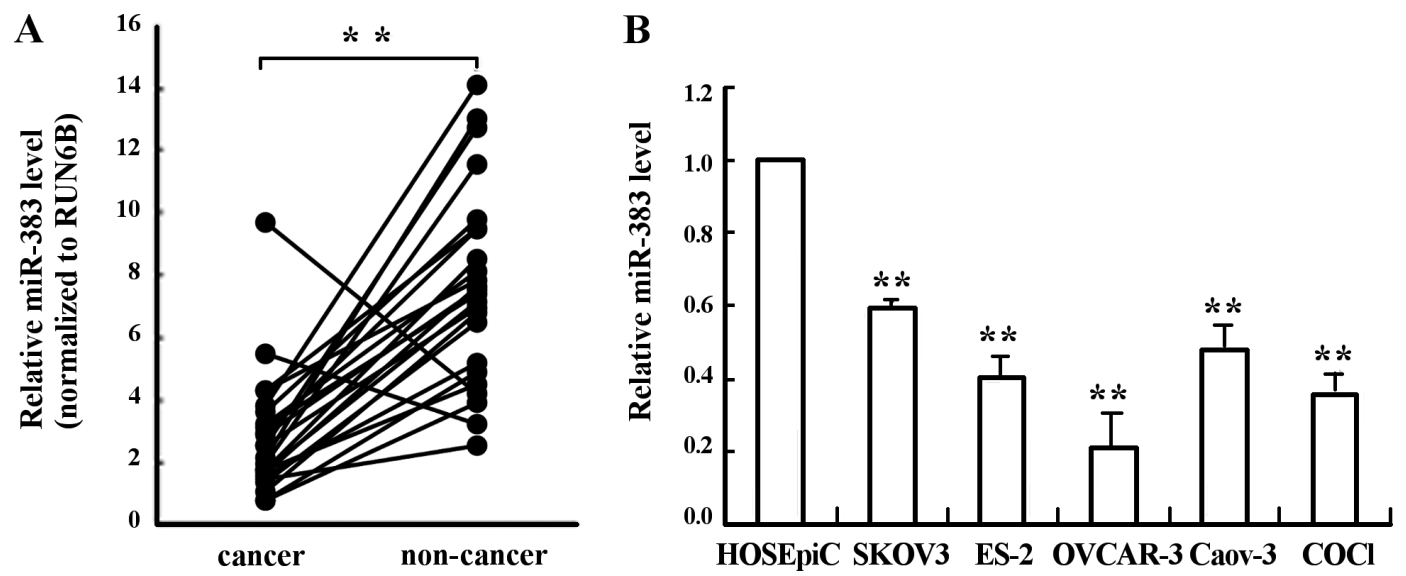

Figure 1. miR-383 was downregulated in ovarian cancer tissues and cell lines. (A) The mean expression level of miR-383 in ovarian cancer tissues was significantly lower than that in pair-matched adjacent noncancerous normal tissues (B) miR-383 was significantly decreased in five ovarian cancer cell lines compared with that in HOSEpiC cell. ${ }^{\star} \mathrm{P}<0.05,{ }^{\star *} \mathrm{P}<0.01$ compared with control group. 
in ovarian cancer, we examined the expression of miR-383 in 24 pairs of ovarian cancer tissues and their matched adjacent normal tissues using qRT-PCR. We found that miR-383 expression was significantly lower in human ovarian cancers than that of their normal counterparts (Figure 1A). Then, we further examined the expression of miR-383 in five different human ovarian cancer cell lines as well as the normal ovarian surface epithelial cell line (HOSEpiC). The results showed a significant downregulation in expression of miR-383 in ovarian cancer lines as compared with HOSEpiC (Figure 1B). SKOV3 and OVCAR3 cells exhibited the highest and lowest expression of miR-383 among ovarian cancer cell lines, respectively, and were selected for further studies.

MiR-383 exhibits the inhibitory effect on ovarian cancer cell proliferation and invasion. To explore the functions of miR-383 in ovarian cancer, we performed knockdown and overexpression experiments in ovarian cancer cells. SKOV3 cells expressing relatively high level of miR-383 and OVCAR3 cells expressing relatively low level of miR-383 were transfected with miR-383 inhibitor and miR-383 mimics, respectively, which decreased the level of miR-383 in SKOV3 by 4.71 folds and increased that in OVCAR3 by 20.5 folds, as compared
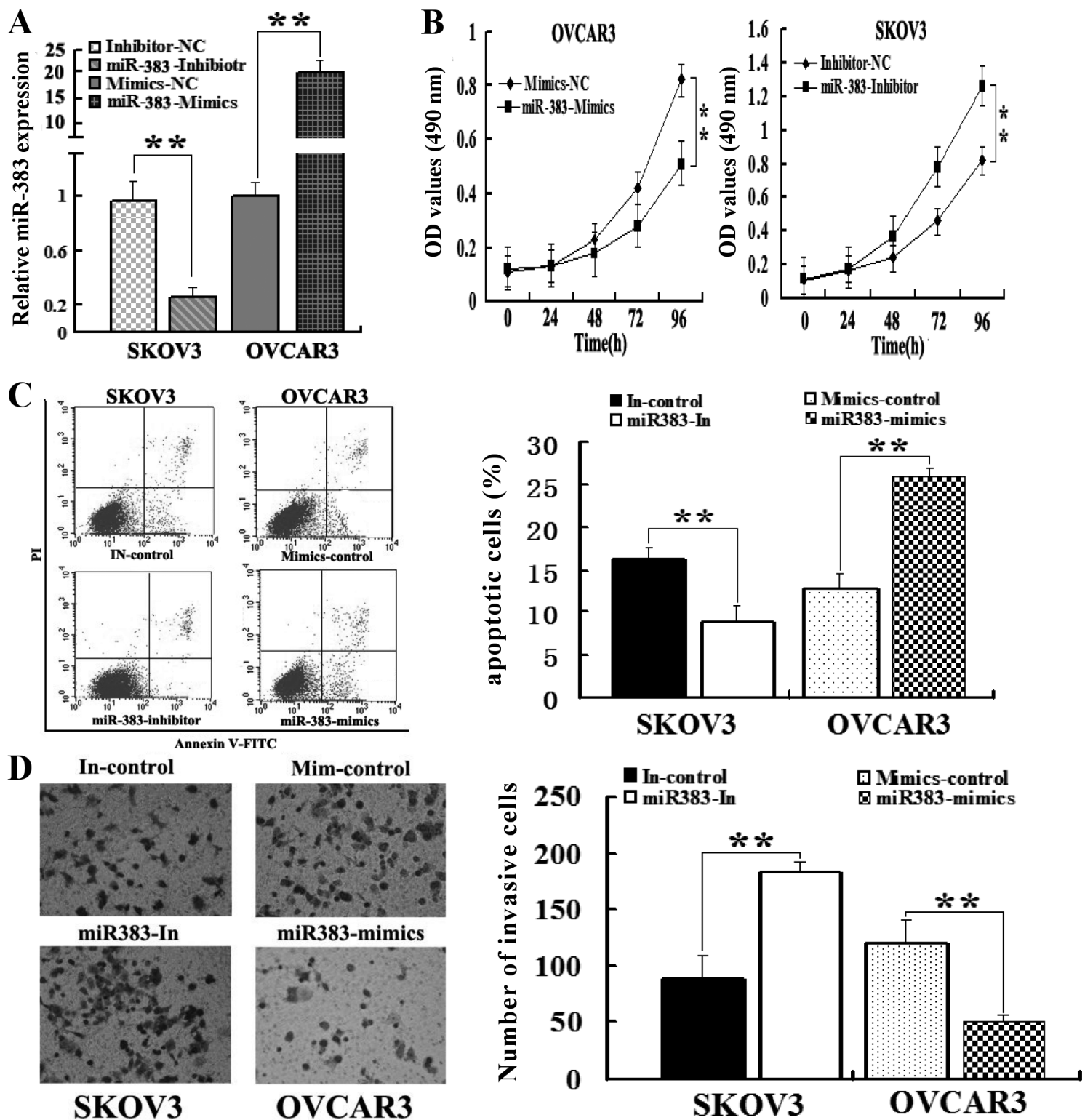

Figure 2. miR-383 suppresses ovarain cancer cell proliferation and invasion. (A) qRT-PCR analysis of miR-383 levels in SKOV3 and OVCAR3 cells, transfected with miR-383 inhibitor and miR-383 mimics, respectively. (B) Determination of SKOV3 and OVCAR3 cells proliferation with MTT assay. (C) Determination of SKOV3 and OVCAR3 cell apoptosis with Flow cytometry. (D) Determination of SKOV3 and OVCAR3 cell invasion with Transwell invasion assay. ${ }^{\star} \mathrm{P}<0.05,{ }^{*} \mathrm{P}<0.01$ compared with control group. 
with corresponding negative control (Figure 2A). MTT assay showed that downregulation of miR-383 significantly increased cell proliferation of SKOV3 cells while upregulation of miR-383 significantly decreased cell proliferation of OVCAR3 cells, as compared with corresponding negative controls (Figure 2B). To further determine the mechanisms of miR-383 influencing ovarian cancer cell proliferation, we analyzed the apoptosis and cell cycle in the treated SKOV3 and OVCAR 3 cells by flow cytometry. We found that the downregulation of miR-383 significantly decreased cell apoptosis rate of SKOV3 cells while upregulation of miR-383 significantly increased cell apoptosis rate of OVCAR3 cells, as compared with corresponding negative controls (Figure 2C). There were no significant changes in the cell cycle for both cell lines (data not shown). These results suggest that miR-383 induced ovarian cancer cell proliferation inhibition was, at least in part, caused by apoptosis.

To investigate the effect of miR-383 on the invasion of ovarian cancer cells, in vitro invasion assays were performed. Matrigel Transwell assays showed that miR-383 markedly suppressed the invasive ability of OVCAR3 cells while antimiR-383 promoted this activity of SKOV3 cells (Figure 2D).

LDHA is a direct target of miR-383. To identify potential targets for miR-383, we used four bioinformatics algorithms: TargetScan, pictar, E1MMo2 and miRanda. All of these approaches predicted LDHA as a target of miR-383, and the 3'-UTR of LDHA mRNA contains a highly conserved binding site from position 80 to 86 for miR-383 seed sequence (the core sequence that encompasses the first 2-8 bases of the mature miRNA, Figure 3A). To confirm whether LDHA was the target of miR-383, we performed Western blot analysis. The level of LDHA protein was significantly decreased by miR-383 overexpression in OVCAR3 cells but significantly increased by silencing of miR-383 in SKOV3 cells (Figure $3 B)$. To further explore whether miR-383 could degrade LDHA mRNA, we performed qRT-PCR. Consistent with the result of Western blot analysis, we found that mRNA level of LDHA was significantly down-regulated by miR-383 overexpression in OVCAR3 cells but up-regulated by depleting of miR-383 in SKOV3 cells (Figure S1). To investigate whether
A

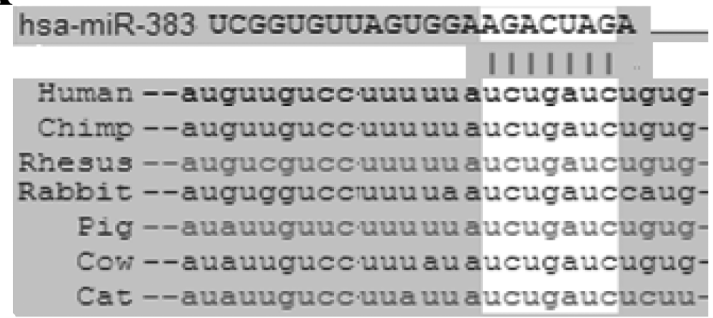

C

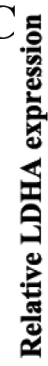

B

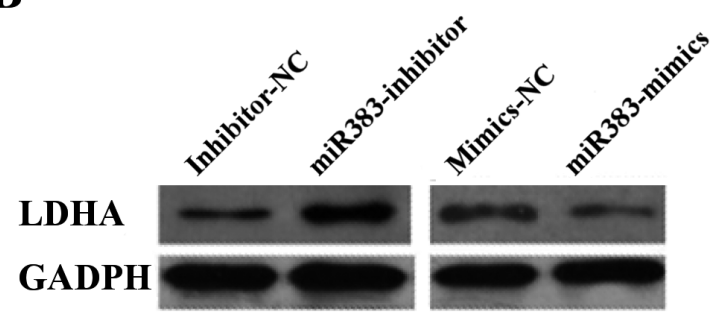

D

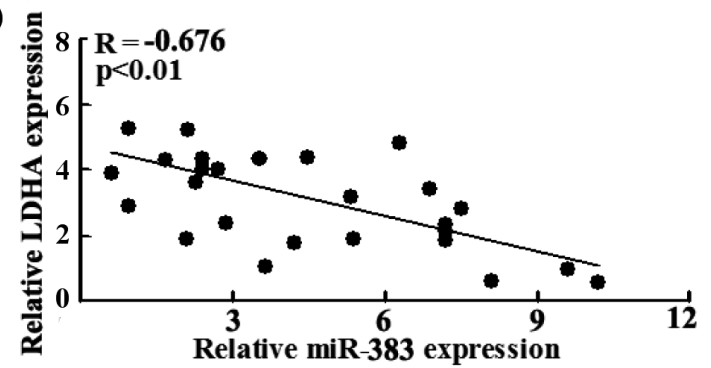

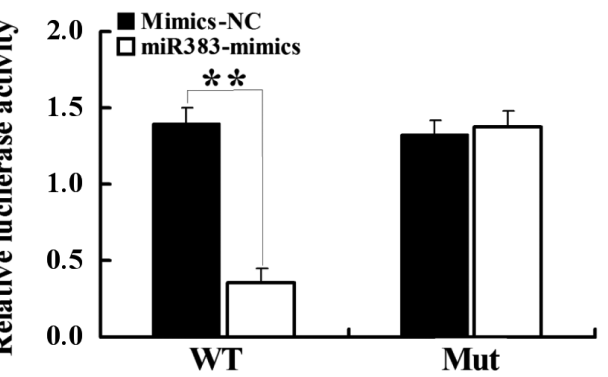

Figure 3. LDHA is a direct target of miR-383. (A) A putative miR-383 binding site in 3'UTR region of LDHA and interspecies conservation of seed matching squences. Mutation was generated in the LDHA 3'UTR by deleting seed matching sequence. (B) Protein level of LDHA was detected by Western blot in SKOV3 and OVCAR3 cells transfected with miR-383 inhibitor and miR-383 mimics along with corresponding controls, respectively. (C) LDHA mRNA level was examined by qRT-PCR and, it was remarkably decreased in ovarian cancer tissues. (D) LDHA mRNA level was inversely correlated with miR-383 level in ovarian cancer tissues (Spearman's correlation analysis). (E) HEK293 cells were co-transfected with miR-383 and WT or Mut LDHA $3^{\prime}$ UTR luciferase reporter construct. ${ }^{\star} \mathrm{P}<0.05,{ }^{\star \star} \mathrm{P}<0.01$ compared with control group. 
the regulations described above in ovarian cancer cell lines are also clinically relevant, we further examined the expression of LDHA mRNA in 24 pairs of ovarian cancer tissues and their matched adjacent normal tissues as previously described. Results indicated that LDHA mRNA was significantly upregulated in ovarian cancer tissues compared with the paired-adjacent normal tissues and negatively correlated with miR-383 level in ovarian cancer tissues (Figure 3C, 3D). This finding was further supported by Western blot analysis which indicated that the protein levels of LDHA were significantly increased in ovarian cancer tissues relative to the matched adjacent normal tissues (Figure S2). To further confirm that whether LDHA is a direct target gene of miR-383, we constructed luciferase reporter vector containing wild-type LDHA 3'UTR with miR-383 binding site(WT) or containing the mutant 3'UTR(deletion of the putative miR-383 target site, MUT). Luciferase activity assay showed that miR-383 significantly inhibited the luciferase activity of LDHA-3'UTR-WT reporter, compared with control, but did not influence the mutant reporter in HEK293 cells (Figure
3E). Together, these data suggested that LDHA was a direct target of miR-383 in ovarian cancer.

MiR-383 suppresses glycolysis in ovarian cancer cells. Given the critical role of LDHA in cancer metabolism, we further investigated the biological function of miR-383 in ovarian cancer glycolysis. We found that overexpression of miR-383 mimics led to a decrease in extracellular lactate $(\mathrm{P}<0.01)$, glucose consumption $(\mathrm{P}<0.01)$ and ATP levels $(\mathrm{P}<0.01)$ in OVCAR3 cells (Figure 4A-C). Furthermore, suppression of the glycolytic phenotype was accompanied by a metabolic shift towards mitochondrial respiration indicated by an increase in the oxygen consumption rate $(\mathrm{P}<0.01)$ (Figure 4D). But when we knocked down the miR-383 expression in SKOV3 cells, we found an increase in extracellular lactate, ATP levels and glucose consumption and a decreased in the oxygen consumption in SKOV3 cells. These results suggested that miR-383 plays vital roles in aerobic glycolysis in ovarian cancer cells.

LDHA mediates miR-383-induced effects on ovarian cancer proliferation, invasion and aerobic glycolysis. We
A

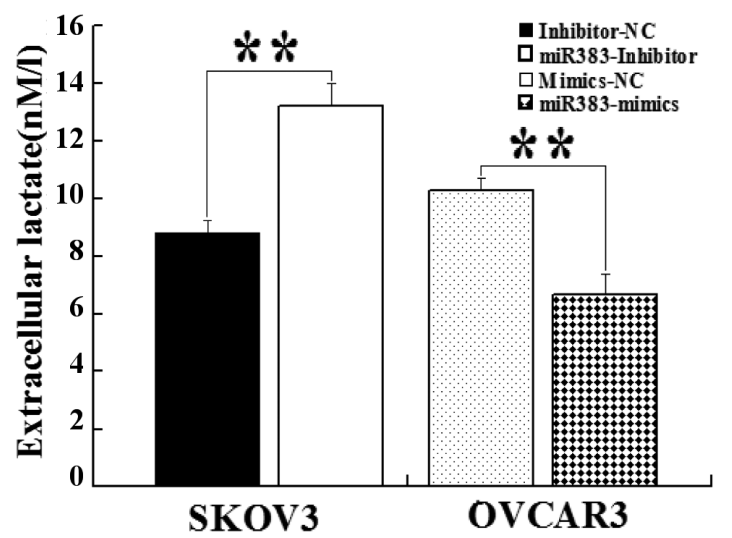

C

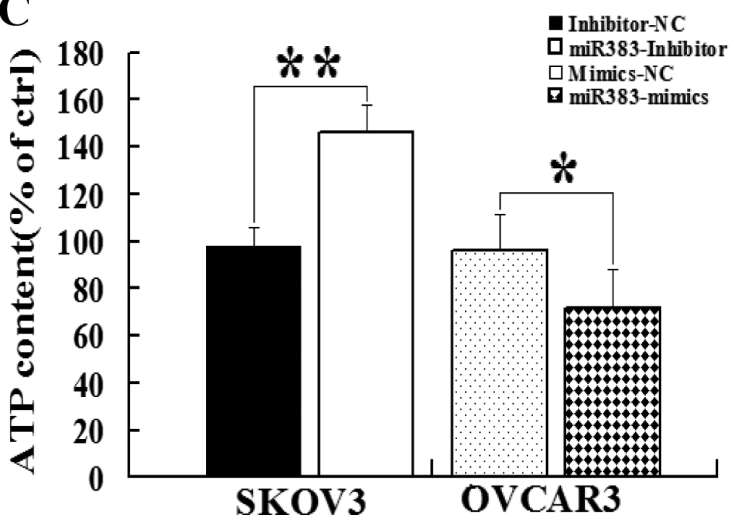

B

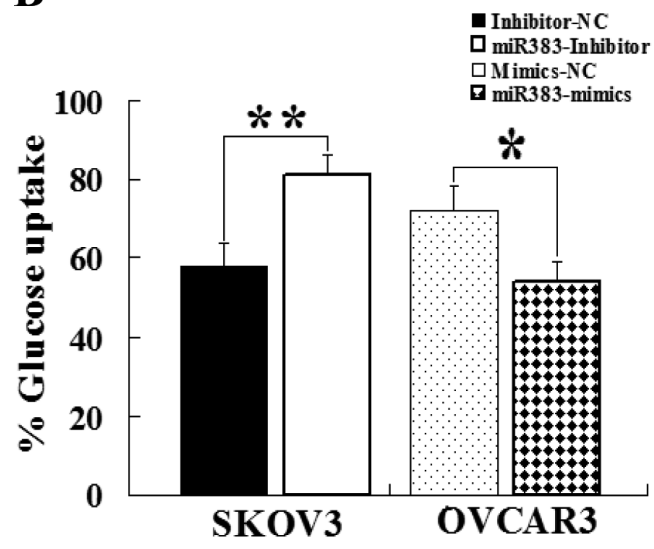

D

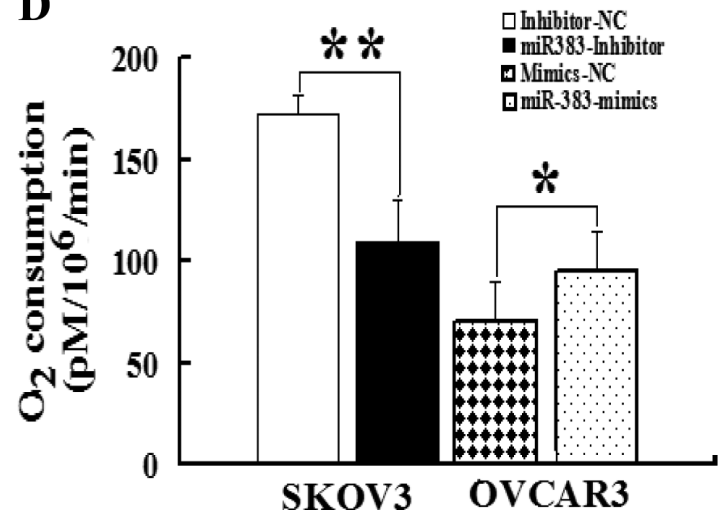

Figure 4. Effect of miR-383 on the metabolism phenotype in ovarian cancer cells. (A) Inhibition of miR-383 expression leads to increased lactate production in SKOV3 cells, and overexpression of miR-383 causes decreased lactate production in OVCAR2 cells. (B) Inhibition of miR-383 expression leads to increased glucose uptake in SKOV3 cells, and overexpression of miR-383 causes decreased glucose uptake in OVCAR2 cells. (C) Inhibition of miR-383 expression leads to increased ATP level in SKOV3 cells, and overexpression of miR-383 causes decreased ATP level in OVCAR2 cells. (D) Inhibition of miR-383 expression leads to decreased oxygen consumption in SKOV3 cells, and overexpression of miR-383 causes increased oxygen consumption in OVCAR2 cells. ${ }^{\star} \mathrm{P}<0.05,{ }^{* *} \mathrm{P}<0.01$ compared with control group. 

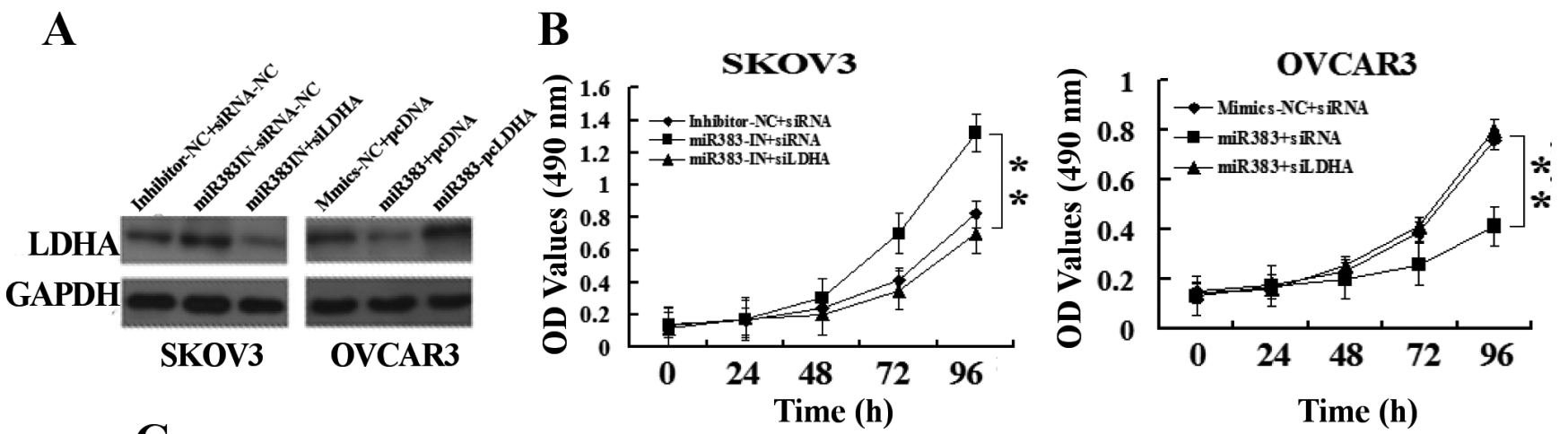

C
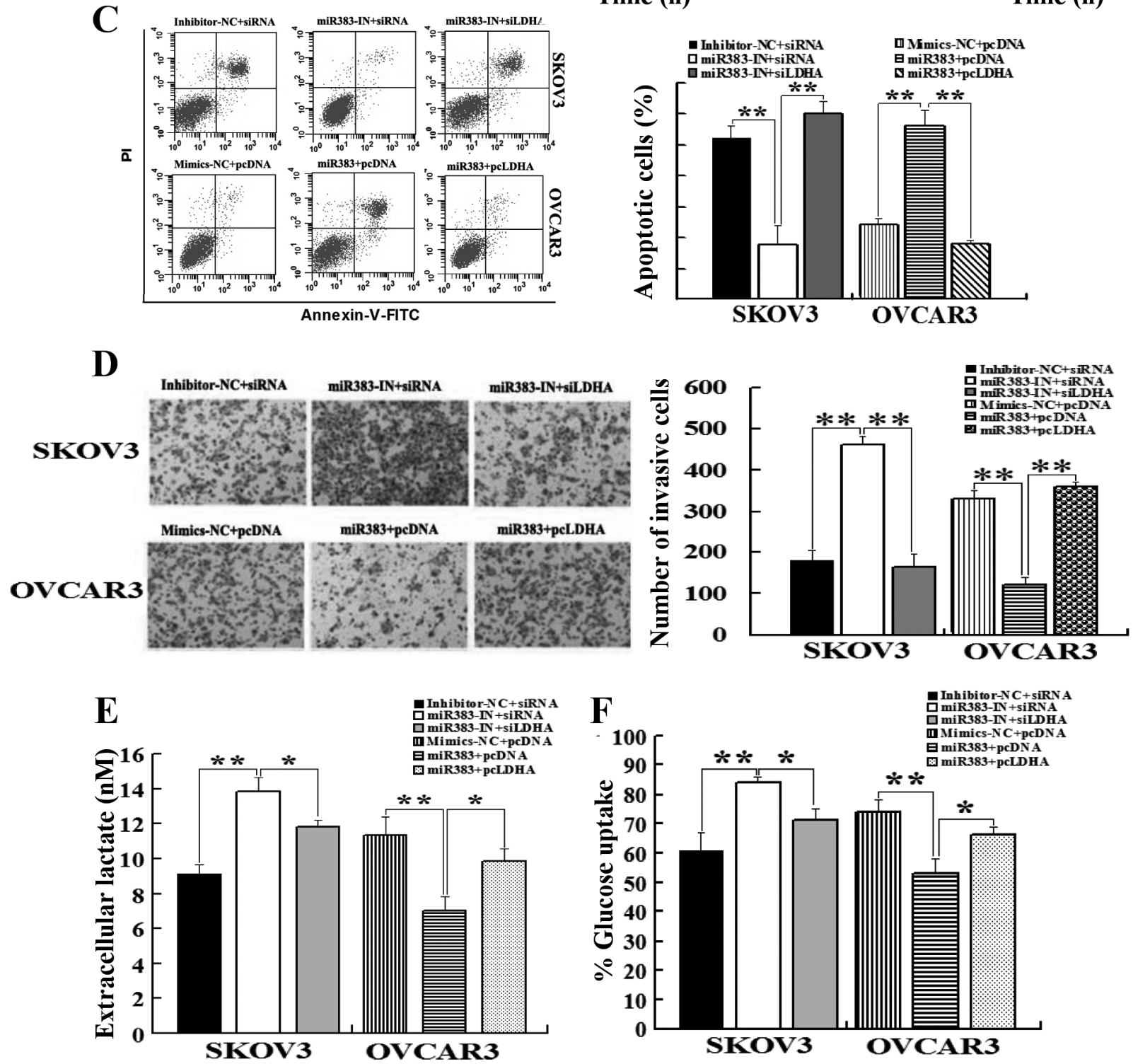

Figure 5. LDHA is involved in miR-383 regulation of ovarian cancer cell proliferation, invasion and aerobic glycolysis. SKOV3 cells were transfected with miR-383 inhibitor or cotransfected with miR-383 inhibitor and LDHA specific siRNAs (siLDHA), while OVCAR3 cells were transfected with miR-383 mimics or cotransfected with miR-383 mimics and LDHA expression vector (pcLDHA). (A) Western blotting analysis of LDHA expression in SKOV3 and OVCAR3 cells. (B) Determination of cell proliferation with the MTT assay. (C) Flow cytometry analysis of the cell apoptosis. (D) Determination of cell invasion ability with the Transwell assay. (E) LDHA reverses miR-383-induced decreases in lactate production. (F)LDHA reverses miR-383-induced decreases in glucose uptake. ${ }^{\star} \mathrm{P}<0.05,{ }^{* *} \mathrm{P}<0.01$ compared with control group. 
further investigated whether LDHA is involved in the tumor suppressor effects of miR-383 on ovarian cancer cells. For this purpose, OVCAR3 cells were cotransfected with both miR-383 mimics and LDHA-expressing vector, and SKOV3 cells were cotransfected with both miR-383 inhibitor and siLDHA. MTT and in vitro invasion assays showed that overexpression of LDHA significantly reversed miR-383-induced suppression of proliferation and invasion of OVCAR3 cells, while silencing of LDHA significantly abrogated protumoral effects of miR-383 inhibitor on the proliferation and invasion of SKOV3 cells (Figure 5A-D).

Next, we investigated whether miR-383 could affect ovarian cancer cell metabolism by targeting LDHA. We found that overexpression of LDHA markedly impaired the miR-383 suppression effects on aerobic glycolysis, while downregulating LDHA expression reversed the effect of miR-383 inhibitor on aerobic glycolysis (Figure 5E, 5F). The results supported the miR-383 and LDHA regulatory relationship on cancer metabolism.

\section{Discussion}

Emerging data has indicated that miR-383 plays a critical role in tumorigenesis as a tumor suppressor. Recent studies have shown that miR-383 is involved in the ovarian follicular and luteal development $[12,13]$. However, few researches have been focused on the relationship between dysregulation of miR-383 and ovarian carcinogenesis.

In a previous miRNAs microarray-based study, miR-383 was found to be downregulated in ovarian cancer tissues compared with matched adjacent normal ovarian tissues[14]. In the present study, we further validated that miR-383 was significantly downregulated in the most of ovarian cancer specimens compared with the adjacent non-tumor tissue. Moreover, we found that ectopic overexpression of miR383 dramatically suppressed proliferation and invasion in OVCAR3 cells, while downregulation of miR-383 promoted proliferation and invasion in SKOV3 cells. This is the first report about the regulatory functions of miR-383 in ovarian cancer proliferation and invasion. However, its roles in vivo and the relationship between deregulation of its expression and clinical outcomes await further studies.

MiRNAs were reported to control gene expression by binding to the 3'-UTR of target mRNAs and either inhibiting translation or inducing degradation of mRNAs. In this study, we confirmed that miR-383 inhibits the expression of LDHA in ovarian cancer cells and luciferase reporter assay confirmed that LDHA is a direct target of miR-383. Furthermore, we found that LDHA expression was greatly upregulated in ovarian cancer tissues and cell lines and our correlation assay showed that the expression levels of miR-383 are negatively associated with those of LDHA mRNA in clinical ovarian cancer tissues. Increased aerobic glycolysis in cancer cells is a universal feature of cancer progression, which increases glucose uptake and metabolic intermediates for macromolecule biosynthesis to support rapid cell growth [26, 27]. LDHA is a key enzyme of the aerobic glycolysis of the cancer cells which favored catalysis of the conversion of pyruvate to lactate. Consistent with these previous reports, our study found that modulating expression of miR-383 could affect the glycolysis in ovarian cancer cells. LDHA has been reported to overexpress in a series of cancer, including ovarian cancer, and promoted cancer development and progression. However, the expression and the mechanism of elevated expression of LDHA in ovarian cancer have not been demonstrated. Our findings provided strong evidence that downregulation of miR-383 may be a potential mechanism for the increase of LDHA expression in ovarian cancer.

Our biological function studies suggested that miR-383 inhibits ovarian cancer proliferation and invasion mainly by targeting LDHA. Moreover, our study has found that miR-383 can inhibit the aerobic glycolysis in ovarian cancer cells through directly targeting LDHA. Our results are in agreement with previous studies which showed that knockdown of the expression of LDHA suppressed cell growth and migration of several cancer cells in vitro [28-30]. These data reveal that miR-383 is critical in shifting the ovarian cancer metabolism and regulating the proliferation and invasion potential of ovarian cancer cell. Therefore, our findings might provide a potential diagnostic marker and therapeutic target for ovarian cancer.

Supplementary information is available in the online version of the paper.

Acknowledgements: This research was supported by the Chinese National Natural Science Foundation Grant No. 81501427 and No. 81370337.

\section{References}

[1] SIEGEL R, WARD E, BRAWLEY O, JEMAL A. Cancer statistics, 2011: The impact of eliminating socioeconomic and racial disparities on premature cancer deaths. CA Cancer J Clin 2011; 61: 212-236. https://doi.org/10.3322/ caac. 20121

[2] SHAPIRA I, OSWALD M, LOVECCHIO J, KHALILI H, MENZIN A et al. Circulating biomarkers for detection of ovarian cancer and predicting cancer outcomes. Br J Cancer 2014; 110: 976-983. https://doi.org/10.1038/bjc.2013.795

[3] LING H, FABBRI M, CALIN GA. MicroRNAs and other noncoding RNAs as targets for anticancer drug development. Nat Rev Drug Discov 2013; 12: 847-865. https://doi.org/10.1038/ $\underline{\operatorname{nrd} 4140}$

[4] LU J, GETZ G, MISKA EA, ALVAREZ-SAAVEDRA E, LAMB J et al. MicroRNA expression profiles classify human cancers. Nature 2005; 435: 834-838. https://doi.org/10.1038/ nature 03702

[5] SUN TY, XIE HJ, HE H, LI Z, KONG LF. miR-26a inhibits the proliferation of ovarian cancer cells via regulating CDC6 expression. Am J Transl Res 2016; 8: 1037-1046. 
[6] MENG X, MULLER V, MILDE-LANGOSCH K, TRILLSCH F, PANTEL K et al. Diagnostic and prognostic relevance of circulating exosomal miR-373, miR-200a, miR-200b and miR-200c in patients with epithelial ovarian cancer. Oncotarget 2016; 7: 16923-16935. https://doi.org/10.18632/ oncotarget.7850

[7] ZHENG HB, ZHENG XG, LIU BP. miRNA-101 inhibits ovarian cancer cells proliferation and invasion by downregulating expression of SOCS-2. Int J Clin Exp Med 2015; 8: 20263-20270.

[8] WANG X, REN Y, WANG Z, XIONG X, HAN S et al. Downregulation of $5 \mathrm{~S}$ rRNA by miR-150 and miR-383 enhances c-Myc-rpL11 interaction and inhibits proliferation of esophageal squamous carcinoma cells. FEBS Lett 2015; 589: 3989-3997. https://doi.org/10.1016/j.febslet.2015.11.012

[9] CHEN L, GUAN H, GU C, CAO Y, SHAO J et al. miR-383 inhibits hepatocellular carcinoma cell proliferation via targeting APRIL. Tumour Biol 2016; 37: 2497-2507. https://doi. org/10.1007/s13277-015-4071-1

[10] HAN S, CAO C, TANG T, LU C, XU J et al. ROBO3 promotes growth and metastasis of pancreatic carcinoma. Cancer Lett 2015; 366: 61-70. https://doi.org/10.1016/j. canlet.2015.06.004

[11] XU D, MA P, GAO G, GUI Y, NIU X et al. MicroRNA-383 expression regulates proliferation, migration, invasion, and apoptosis in human glioma cells. Tumour Biol 2015; 36: 7743-7753. https://doi.org/10.1007/s13277-015-3378-2

[12] DONADEU FX, SCHAUER SN. Differential miRNA expression between equine ovulatory and anovulatory follicles. Domest Anim Endocrinol 2013; 45: 122-125. https://doi. org/10.1016/j.domaniend.2013.06.006

[13] DONADEU FX, SCHAUER SN, SONTAKKE SD. Involvement of miRNAs in ovarian follicular and luteal development. J Endocrinol 2012; 215: 323-334. https://doi.org/10.1530/ JOE-12-0252

[14] VILMING ELGAAEN B, OLSTAD OK, HAUG KB, BRUSLETTO B, SANDVIK L et al. Global miRNA expression analysis of serous and clear cell ovarian carcinomas identifies differentially expressed miRNAs including miR-200c-3p as a prognostic marker. BMC Cancer 2014; 14: 80. https://doi. org/10.1186/1471-2407-14-80

[15] VANDER HEIDEN MG, CANTLEY LC, THOMPSON CB. Understanding the Warburg effect: the metabolic requirements of cell proliferation. Science 2009; 324: 1029-1033. https://doi.org/10.1126/science.1160809

[16] WARBURG O. On the origin of cancer cells. Science 1956; 123: 309-314. https://doi.org/10.1126/science.123.3191.309

[17] ZHAO D, ZOU SW, LIU Y, ZHOU X., MO Y et al. Lysine-5 acetylation negatively regulates lactate dehydrogenase $\mathrm{A}$ and is decreased in pancreatic cancer. Cancer Cell 2013; 23: 464-476. https://doi.org/10.1016/j.ccr.2013.02.005
[18] ZHANG R, SU J, XUE SL, YANG H, JU LL et al. HPV E6/p53 mediated down-regulation of miR-34a inhibits Warburg effect through targeting LDHA in cervical cancer. Am J Cancer Res 2016; 6: 312-320.

[19] JIANG W, ZHOU F, LI N, LI Q, WANG L. FOXM1-LDHA signaling promoted gastric cancer glycolytic phenotype and progression. Int J Clin Exp Pathol 2015; 8: 6756-6763.

[20] WANG J, WANG H, LIU A, FANG C, HAO J et al. Lactate dehydrogenase A negatively regulated by miRNAs promotes aerobic glycolysis and is increased in colorectal cancer. Oncotarget 2015; 6: 19456-19468. https://doi.org/10.18632/ oncotarget. 3318

[21] WANG X, DUAN W, LI X, LIU J, LI D et al. PTTG regulates the metabolic switch of ovarian cancer cells via the c-myc pathway. Oncotarget 2015; 6: 40959-40969.

[22] QIU H, JACKSON AL, KILGORE JE, ZHONG Y, CHAN LL et al. JQ1 suppresses tumor growth through downregulating LDHA in ovarian cancer. Oncotarget 2015; 6: 6915-6930. https://doi.org/10.18632/oncotarget.3126

[23] MONDAL S, ROY D, CAMACHO-PEREIRA J, KHURANA A, CHINI E et al. HSulf-1 deficiency dictates a metabolic reprograming of glycolysis and TCA cycle in ovarian cancer. Oncotarget 2015; 6: 33705-33719.

[24] WANG N, ZHOU Y, ZHENG L, LI H. MiR-31 is an independent prognostic factor and functions as an oncomir in cervical cancer via targeting ARID1A. Gynecol Oncol 2014; 134: 129-137. https://doi.org/10.1016/j.ygyno.2014.04.047

[25] WANG N, ZHAN T, KE T, HUANG X, KE D et al. Increased expression of RRM2 by human papillomavirus E7 oncoprotein promotes angiogenesis in cervical cancer. Br J Cancer 2014; 110: 1034-1044. https://doi.org/10.1038/bjc.2013.817

[26] GATENBY RA, GILLIES RJ. Why do cancers have high aerobic glycolysis? Nat Rev Cancer 2004; 4: 891-899. https://doi. org/10.1038/nrc1478

[27] JONES RG, THOMPSON CB. Tumor suppressors and cell metabolism: a recipe for cancer growth. Gene Dev 2009; 23 : 537-548. https://doi.org/10.1101/gad.1756509

[28] YAO F, ZHAO T, ZHONG C, ZHU J, ZHAO H. LDHA is necessary for the tumorigenicity of esophageal squamous cell carcinoma. Tumour Biol 2013; 34: 25-31. https://doi. org/10.1007/s13277-012-0506-0

[29] SHENG SL, LIU JJ, DAI YH, SUN XG, XIONG XP et al. Knockdown of lactate dehydrogenase A suppresses tumor growth and metastasis of human hepatocellular carcinoma. FEBS J 2012; 279: 3898-3910. https://doi.org/10.1111/j.17424658.2012.08748.x

[30] LE A, COOPER CR, GOUW AM, DINAVAHI R, MAITRA A et al. Inhibition of lactate dehydrogenase A induces oxidative stress and inhibits tumor progression. Proc Natl Acad Sci U S A 2010; 107: 2037-2042. https://doi.org/10.1073/ pnas.0914433107 


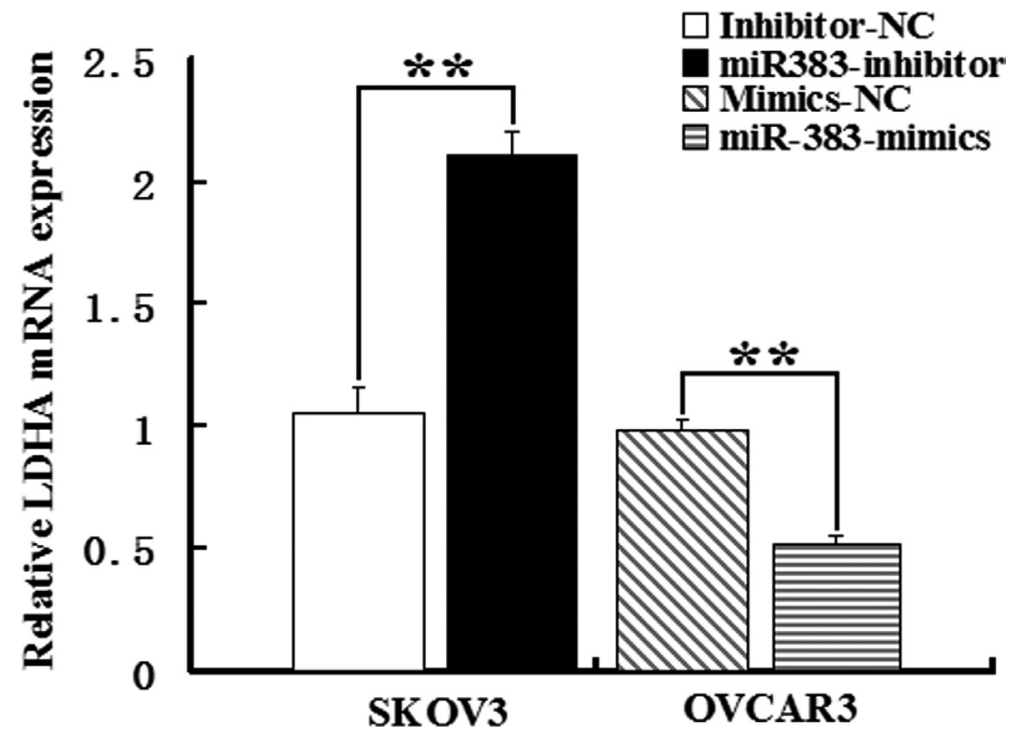

Supplemental Figure 1. LDHA mRNA expression was determined by qRT-PCR in SKOV3 and OVCAR3 cells transfected with miR-383 inhibitor and miR383 mimics along with corresponding controls, respectively. The results indicated that miR-383 could degrade LDHA mRNA in ovarian cancer cells.

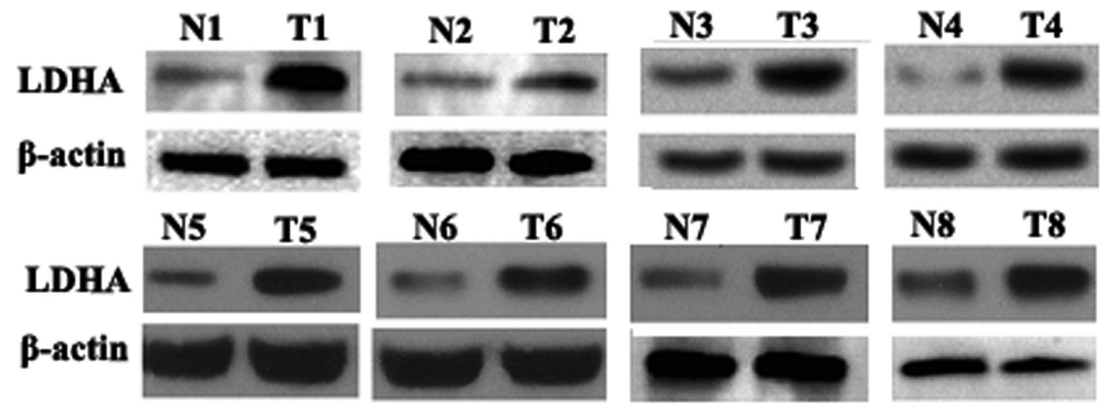

Supplemental Figure 2. 8 paired ovarian cancer tumor tissues $(\mathrm{T})$ and adjacent normal tissues $(\mathrm{N})$ were randomly selected from 24 patients to test LDHA protein expression by Western blotting. The results demonstrated that the level of LDHA protein was markedly increased in ovarian cancer tumor tissues compared with neighboring normal tissues. 